\title{
Unlicensed Personnel's Experience of Digital Signing for Medication Administration in Municipal Healthcare
}

\author{
Pennbrant Sandra ${ }^{\circledR}$, Karlsson Christina, Karlsson Margareta \\ Department of Health Sciences, University West, Trollhättan, Sweden \\ Email: margareta.karlsson@hv.se, sandra.pennbrant@hv.se, christina.karlsson@hv.se
}

How to cite this paper: Sandra, P., Christina, K. and Margareta, K. (2020) Unlicensed Personnel's Experience of Digital Signing for Medication Administration in Municipal Healthcare. Open Journal of Nursing, 10, 1163-1177.

https://doi.org/10.4236/ojn.2020.1012083

Received: November 2, 2020

Accepted: December 7, 2020

Published: December 10, 2020

Copyright ( 2020 by author(s) and Scientific Research Publishing Inc. This work is licensed under the Creative Commons Attribution International License (CC BY 4.0).

http://creativecommons.org/licenses/by/4.0/

\begin{abstract}
Aim: To describe unlicensed personnel's experience of digital signing lists via a smartphone application for the distribution of medication in municipal healthcare in Western Sweden. Design: A qualitative and quantitative design was used. Methods: The study included 48 unlicensed personnel, 28 of whom answered an open-ended questionnaire, while an additional 20 volunteered for individual interviews. The material was analysed by qualitative content analysis. Results: The results indicate that digital signing lists via a smartphone application are feasible, and efficient and facilitate the work. However, some aspects negatively affected the sense of security, meetings with patients and quality of care, such as an insufficient internet signal in some rural areas, difficulty remembering the password, as well as the change of focus from patient to smartphone. To improve quality of care and the meeting with the patient, it is crucial that the technology works and that unlicensed personnel develop technical skills.
\end{abstract}

\section{Keywords}

Digital Signing, eHealth, Municipal Healthcare, Patient Safety,

Quality of Care, Unlicensed Personnel, Welfare Technology

\section{Introduction}

Welfare technology is becoming increasingly common in healthcare both internationally and nationally [1]. In Sweden, older persons with complex ill-health who are living at home are supported by unlicensed personnel (UP) from the municipal healthcare organization in the administration of their medication and some municipal healthcare organizations have implemented a 
digital signing list. The handling of medication is delegated to UP and to ensure patient safety that they must sign a medication list when they have administered the patient's medication. While welfare technology should contribute to a good and sustainable everyday life as well as quality care for patients, it should also provide increased security and independence for UP [2]. As many older persons in municipal healthcare are supported by UP for the administration of their medication via the digital signing list, it is important to gain more knowledge about use of the technology, its possibilities, and limitations. With increasing technology in healthcare, it is also essential to gain more knowledge about the UP's experiences of digital signing lists via a smartphone application for distribution of medication in a municipal healthcare organization in Western Sweden. Research in this area is limited and the present study will contribute relevant knowledge.

\section{Background}

In Sweden, delegation of responsibility for handling medication is regulated by the Regulations and General Advice of the National Board of Health and Welfare [3]. According to the Patient Safety Act [4], delegation of responsibility may only take place if it is compatible with good and safe care and if there are guidelines on how to delegate. Registered nurses (RN) in the municipal healthcare organization are responsible for delegating the responsibility for handling medication in patients' own homes to UP. Previous research shows that delegation can reduce the RNs' workload [5]. Although UP consider delegation to be part of their work, they do not feel familiar with it.

In Sweden, eHealth and welfare technology has developed most in the healthcare sector for older people [1]. There are several advantages of digital signing lists, such as increased patient safety and a clearer overview of patient needs, making it easier for staff to report and follow-up cases [6], in addition to increased information between stakeholders, less stress and more efficient care [7]. In the study on eHealth in Swedish homecare nursing [8], the digital medication list was described as increasing the implementation rate, while mobile documentation was the most popular type of eHealth. According to Skär \& Söderberg [9], implementation of eHealth services involves ethical challenges. Therefore, to provide high-quality care eHealth services should be developed and implemented on the basis of the patient's needs and in consultation with UP. When implementing and using digital technology, it is important to be aware of the fact that the role of personnel is changing and ensure that there are sufficient resources to support person-centred care [10]. According to Nakrem et al. [11], the relationship between patient and caregiver may suffer if the technology does not meet patients' needs and digital services are not safe and reliable.

Findings reveal the richness, diversity and potential of eHealth for enabling safer, more efficient and patient-centred healthcare. Patient safety is a basic quality dimension in healthcare, which means that easily accessible and high quality 
care that should be based on respect for the patient's self-determination and integrity, as well as meet her/his need for continuity and safety [12]. Patient safety involves protecting the patient against health damage, which means that patients should not be harmed in connection with healthcare measures or that care does not fail to implement the measures that are justified based on the patient's condition [4] [13]. Patient safety is a multidimensional concept that includes environmental, human and individual characteristics that affect behaviour and performance in practice, which in turn have an impact on well-being, health and safety [14]. According to Khalil \& Lee [15], reporting and documentation are barriers to medication safety. Metlay et al. [16] demonstrate that older persons do not remember whether they had received instructions about their medication and do not use existing digital systems to help them organize it. UP in municipal care who are delegated to administer medication to older persons in their own home are responsible for doing so in a patient-safe way. When introducing digital signing lists for medication in the municipal healthcare organization, it is necessary to examine the conditions and implementation experience of UP, who must be delegated to administer medication to older persons in their home by an $\mathrm{RN}$.

The use of digital signing lists via smartphone application in healthcare is increasing and previous studies have shown that the medication safety process is complex and contains many elements from prescription to administration and medicine monitoring [5] [15] [16]. Although studies have been conducted in the area of digital signing via smartphone in healthcare, few have focused on how UP experience digital signing lists via a smartphone application in municipal healthcare. Therefore, experience of digital signing lists via a smartphone application in municipal healthcare on the part of UP is an important factor to be considered in the context of patient safety and safer care.

\section{Aim}

The aim of this study was to describe unlicensed personnel's experience of digital signing lists via a smartphone application for the distribution of medication in municipal healthcare in Western Sweden.

\subsection{Design}

An open-ended questionnaire and individual interviews were the methods chosen to enable UP to share their experience of digital signing lists via a smartphone application for the distribution of medication in municipal healthcare. The study was exploratory and efforts were made to collect as much information as possible in the specific area.

The open-ended questionnaire enabled UP to describe their experiences. Individual interviews provide opportunities for follow-up questions, which can contribute to a deeper understanding of the studied subject [17]. This approach allows the person to express experiences in his/her own words [18]. 


\subsection{Sample and Setting}

The study was conducted in Western Sweden during 2019. In order to recruit participants, a written request with information about the study was sent to five managers with responsibility for UP in the municipality as well as to UP working in health and social care.

Approval was obtained from all five department managers, after which they were sent the open-ended questionnaires for distribution to the UP. Subsequently, information letters were sent to the managers, who distributed them to UP who had agreed to participate in the interview study. Participation in the study was voluntary and the data were treated confidentially [18].

Municipal health and social care is part of primary care, which is the health service that responds to the population's need for basic medical treatment, nursing, prevention and rehabilitation that does not involve the medical and technical resources of hospitals. Municipal healthcare includes home healthcare and rehabilitation, in which UP assist RNs, district nurses, occupational therapists and physiotherapists [19].

The empirical data consisted of two data sets: an open-ended questionnaire answered by 28 UP and individual, semi-structured, follow-up interviews for which 20 additional UP volunteered. The UP were invited to describe their experiences of implementation and use of digital signing.

Of the 28 UP who answered the questionnaire, 25 were women and 3 were men (average age $=44$ years; median $=44.5$ years). Their years in the profession ranged from 2 to 52 (average $=16.5$ years; median $=6$ years).

Of the 20 UP who participated in the individual, semi-structured, follow-up interviews, 17 were women and 3 were men (average age $=44$ years; median $=$ 43 years). Their years in the profession ranged from 0 to 42 (average $=15.6$ years; median $=17$ years).

\subsection{Data Collection}

The data collection was conducted during 2019 (the open-ended questionnaire in March-April and the individual, semi-structured, follow-up interviews in October-November). Two of the authors (SP and MK) developed the open-ended questionnaire, which contained the following questions: education in digital signing lists, the use of digital signing lists and skill development needs related to digital signing lists. A pilot study was carried out to ensure that the questions in the questionnaire were clear, easy to understand and relevant to the aim of the study. The pilot study also tested the validity and reliability of the questionnaire, which means that the questions are consistent with the purpose of the survey [20]. The results of the pilot study were processed and no questions in the questionnaire required adjustment. Of the total of 98 questionnaires, 28 were returned, resulting in a response rate of $29 \%$.

One of the researchers (CK) conducted the individual, semi-structured, follow-up interviews with 20 other UP who volunteered to participate. Two of the 
authors (SP and MK) developed the questions for the semi-structured follow-up interviews, which focused on the following issues: approach to medicine and signing, positive and negative aspects of using a digital application and patient safety. The individual interviews lasted 14 to 30 minutes and were conducted at the workplace of the UP. An interview guide was used and the same questions were asked in all interviews, which were audio-taped and transcribed verbatim. The authors checked and corrected the transcription to ensure the quality of the data.

\subsection{Data Analysis}

The open-ended questions in the questionnaire were analysed by means of qualitative content analysis to identify the manifest meaning [17]. First, the authors individually read the participants' answers. Then, the content of each question was compiled to identify patterns, which in turn were divided into categories. Throughout the process the authors carefully compared the identified patterns and categories to achieve a coherent categorization. These categories represent the manifest content.

The text from the individual, semi-structured, follow-up interviews was analysed by means of qualitative content analysis [17]. The analysis took account of both the manifest and the latent content. The analysis of the manifest content focused on the visible and obvious, while the analysis of the latent content led to an interpretation of the underlying message in the text. First, the interview texts were read to gain an overall impression of the material. Meaning units (words, sentences) were identified that corresponded to the aim. In order to make the text more manageable, the meaning units were condensed while retaining their original content. The condensed meaning units were marked and coded. A code is at a descriptive level and can be considered an expression of the manifest content of the text. The codes were then sorted and grouped according to their underlying content, based on similarities and differences. During this part of the analysis, an interpretation is made to try to make visible what is not clear in the interview text (the latent content). This comparative analysis process identified subthemes and subsequently four themes: Education, Work situation, Technology and Care relationship.

An example of the analysis process is presented in Table 1. In order to substantiate the interpretation of the participants' statements, quotations are included in the results to enable the reader to assess their credibility.

The data from responses to the open-ended questions in the questionnaire

Table 1. Examples of the analysis process.

\begin{tabular}{lllr}
\hline Meaning units & Condensed & Code & Subtheme Theme \\
\hline $\begin{array}{llll}\text { Sometimes there is a poor } & \text { Sometimes there is a } & \text { It's hard with a } \\
\text { internet connection. You } & \text { poor internet } & \text { poor internet } & \text { Technology Concerns when } \\
\text { can't see anything. It's very } \\
\text { hard }\end{array}$ & $\begin{array}{l}\text { connection. You can't } \\
\text { see anything }\end{array}$ & & technology fails \\
\end{tabular}


and the individual, semi-structured, follow-up interviews were analysed separately by two of the authors (SP and MK) and subsequently discussed in order to draw conclusions.

\subsection{Trustworthiness}

Preunderstanding refers to the researcher's previous insights and knowledge [18]. Throughout the research process, researchers should reflect on their preunderstanding to enhance their awareness of its potential impact on their work. Preunderstanding may hamper a researcher's work or enhance her/his comprehension and interpretation of the data. In this study, the authors addressed their preunderstanding by maintaining a conscious, critical and reflective approach to it throughout the analysis process.

When qualitative data is being evaluated, the credibility, dependability, confirmability and transferability of the analysis must be considered [17]. To enhance the credibility of the data collection and analysis, the material was collected from various workplaces in the municipality in a manner that ensured variation in the ages and experience of the UP. According to Lundman \& Hällgren Graneheim [17], credibility requires variation. The authors are confident that the participating UP provided a true description of their experiences of digital signing lists using smartphone applications for the distribution of medication, which strengthens the study's credibility. The authors read the data material and together developed the subthemes and themes, thus enhancing the dependability. In the course of the analysis process, the codes, subthemes and themes were compared with the interviews, thus strengthening the confirmability. Moreover, to further enhance the confirmability, the authors related their findings to the results of previous research. According to Lundman \& Hällgren Graneheim [17], a study's transferability may be strengthened by the provision of a clear description of the context, sample selection, inclusion criteria and analysis process. In order to enable readers to gauge the transferability of the present results to similar healthcare settings, they are presented as extensively as possible with relevant quotations.

\subsection{Ethical Considerations}

The regional ethical review board approved the study (Dnr 932-18), which is a prerequisite for ethically acceptable and reliable scientific research in accordance with the Declaration of Helsinki (2018). Information about the study and the open-ended questionnaires were distributed to the participants by the manager. The questionnaires were confidential and the UP returned the completed questionnaire in a sealed envelope to one of the authors.

Before the interview the participants were informed in writing and then orally that participation was voluntary and they had the right to withdraw at any time without giving a reason and without any negative consequences in the workplace, in accordance with the Declaration of Helsinki. Oral and written consent was 
obtained from the participants in connection with the interview. Furthermore, all information was stored confidentially and anonymised.

\section{Results}

The analysis comprised the following four themes: Education, Work situation, Technology and Care relationship. The four themes were based on six subthemes (Table 2).

\subsection{Education}

\subsubsection{Need for Education}

The UP described that the education they received had been inadequate for handling digital signing lists: "Inadequate education in the introduction of digital signing lists" (Questionnaire answer). Most felt that it was a case of "learning by doing" and they taught themselves by using the digital signing application. On the other hand, UP considered that they had received "good support from nurses following the introduction of digital signing lists" (Questionnaire answer). A challenge in the use of digital signing lists was remembering the password for the application and signing out after administering the medication or leaving the patient's home: "To remember and to take the time to sign at the home of the patient" (Questionnaire answer).

The UP described that many steps needed to be performed when signing in to the mobile phone application:

... first I am in an application to see where the patient lives, who the patient is and what the patient needs help with. Then I have to go in and search another application to find the patient's name... (Individual Interview 7)

\subsubsection{Regular Follow-Up}

The UP stated that when something new is implemented it is usually difficult to absorb all the information at once. It is important to understand that learning takes time. Therefore, the participants believed that it would be "good with a few reunions and regular support and follow-up" (Questionnaire answer). The UP felt responsible for the correct updates to the applications but they had not always functioned correctly. When the UP were unsure about how to do it, they often handed over responsibility to another staff member who was more familiar

Table 2. Themes and subthemes.

\begin{tabular}{ll}
\hline Themes & Subthemes \\
\hline Education & - Need for education \\
& - Regular follow-up \\
Work situation & - Safer care \\
& - Technology can provide a warning \\
Technology & - Concerns when technology fails \\
Care relationship & - Lack of quality care in the meeting \\
\hline
\end{tabular}


with it and who they trusted.

...it hasn't worked out so well when it comes to updating the app, it hasn't worked really well... updates are very hard, so you almost do not dare to do it, I don't do it, but hand it over to someone who is an expert on it. (Individual interview 7)

The UP described the need for both technical knowledge and education on how to "force" a program to end.

\subsection{Work Situation}

\subsubsection{Safer Care}

It emerged that digital signing via a smartphone had made the work-situation more secure for the UP and it was easier to see which medication should be given to the older person during the visit. The UP looked in the app a great deal in order not to forget to administer the correct medication and to sign out: "digital signing lists gave an overview and I felt it was safer for the patient that the right drug was given at the right time" (Questionnaire answer). It was also helpful for keeping track of whether patients had received their medication:

... I find it easier to keep track, so that everyone has been given their medication. (Individual interview 19)

The UP experienced that digital signing was positive as it became clearer what medication they should give to the patient. It was also easier to sign a medication in the application at a later stage if the UP had forgotten to do so in the patient's home.

Furthermore, they stated that it was an advantage to be able to sign the application at any time and go into it and check if there was something they were unsure about, which was not as easy to do with paper records:

It is positive that you can go in and see what medication the patient has/.../you can see it at any time. This is not the case with a paper document, which is only in the medicine cabinet at the patient's home. (Individual interview 7)

\subsubsection{The Technology Can Provide a Warning}

The UP described that there was a great deal to keep track of in terms of what would be entered and signed into the application. They stated that if they had forgotten to log out of the application the security function automatically logged them out after about a minute or when they logged in to the next patient. In the following quotation, a UP describes logging in and logging out in the application:

... you are logged out after one minutel..../In order to enter another patient, I need to $\log$ back in. (Individual interview 1)

According to the UP, a particularly difficult situation is when a staff member 
did not remember to sign in to the application, as a red warning light appears indicating that they had forgotten to sign. If a staff member repeatedly forgets to sign and receives many red alerts, she/he might no longer be delegated to administer medication, as described in the following quotation:

... red warning light... comes up as a warning if you forget to sign... if we have many red plops, they (the nurses) no longer delegate us... nurses see it right away... we have been given an exemption for certain medicines if we are late. (Individual interview 18)

...you can see immediately that a red ball shines, for example, that the person should have had medication two hours ago. You call and inform the nurse. It is good for the patient and reduces the risk of errors... (Individual interview 16)

\subsection{Technology}

\section{Concerns When Technology Fails}

The UP pointed out that there was always something that did not work, such as when the network connection was interrupted. When the network did not work, they were allowed to sign a paper list and then contact the nurse, who in turn contacted those responsible for the internet connection in the municipality. The following quotation describes network connectivity issues:

... there is always something malfunctioning, it can be the WiFi, it can be the connection... when it does not work it is a disaster... The nurse must be contacted when it does not work/.../ then someone fixes it so that it works again. (Individual interview 1)

The UP described that although digital signing was considered positive, a prerequisite was that the technology worked: "feel safe and secure due to technology" (Questionnaire answer). However, the network did not always function, which meant additional work for the UP when they had to sign in. The following quotation describes the situation when the network is not working:

Sometimes the internet connection is very poor... You can't see anything... It's very hard... (Individual interview 6)

The UP stated that when network disruption occurred, those in the municipality responsible for the network were usually able to solve the problem fairly quickly. Sometimes it was not the internet connection itself that malfunctioned, but because the reception was poor or non-existent in some of the houses where the patients lived:

... what is bad is when WiFi in the houses where patients live stops working, then the app does not work ... it is not good because you cannot see what medicine the patient should have. (Individual interview 6)

The UP described that it could sometimes be difficult to log in and that the program "froze", which meant that they had to find other ways to get into the 
application. Sometimes they would force the application to stop, turn off the phone and then start it up again:

Sometimes it can be difficult to log in... sometimes it just freezes and crashes and then I have to turn it off and start it up again/.../sometimes I have to go in and force the program to stop and then restart it... can be a bit tedious if you do not really know how to solve the problem.... (Individual interview 4)

\subsection{Care Relationship}

\section{Lack of Quality Care in the Meeting}

It emerged that the UP felt rude when they took out their smartphone to see what medication the patient should have and to sign in each time they administered it when in her/his home: "The patients think that the staff play games on their mobile" (Questionnaire answer). One obstacle is that patients are unable to keep track of which medications they have or should have received: "The patients can't see for themselves if they've been given their medication" (Questionnaire answer). The following quotation describes how UP felt when they were busy with the smartphone:

... sit here and see what $1 \mathrm{~m}$ going to give and the patient sits on the edge of the bed and waits, it feels wrong. But $P$ ve talked to the patients about that, now I sign via the phone, so Pll just check what it is that you should have. But it's a bit sad that you have to have your phone when you' re with the patient. (Individual interview 8)

Although the patients were informed that UP signed for medications via a smartphone application, the shift in focus from patient to phone still did not feel good for the UP.

\section{Discussion}

The aim of this study was to describe UP's experiences of digital signing lists via a smartphone application for the distribution of medication in municipal healthcare in Western Sweden.

The results revealed that technological developments will lead to major challenges in the content of the UP's work. Therefore, UP need education and regular follow-ups to improve their professional skills when introducing digital signing lists. RNs report that rules and regulations are not in line with the unspoken expectation associated with delegating the distribution of medication to UP. To ensure patient safety in the context of delegation, it is vital that nurses follow-up and have good communication with UP [21].

In this study, it emerged that the technology enables the UP to work towards patient safety and quality care. According to De Raeve et al. [22], eHealth increases the ability to provide safer, more efficient and patient-centred healthcare. State Public Reports [2] underline the need for the state to take more responsi- 
bility in supporting municipalities in the expansion of broadband for internet services.

The study shows that the biggest challenge for UP in the implementation of digital signing lists in the municipal healthcare sector was feeling safe and secure when using the technology and that the technology functioned as intended. Technological developments will lead to major changes in the content of work, requiring additional professional skills on the part of employees. In order for the new technology to facilitate work and contribute to increased well-being, better control, mental health and good work performance, all employees need to have an opportunity for continuous competence development [23]. Regardless of the choice of digital service, the intervention should be based on the individual's needs and circumstances and designed together with her/him. It is already difficult to recruit enough competent eldercare staff and this problem will increase in the future. While the provision of skills alone cannot attract new staff, the skills of existing personnel must be retained and enhanced by creating a sustainable and attractive work environment. Through digitization and the use of e-health technology, work can be organized in ways that may give staff more time to care, which in turn can contribute to reducing stress among staff [2]. Therefore, it may be necessary to provide appropriate education for staff to enhance strength and safe care in association with the implementation of digital signing lists.

In order for e-health technologies such as digital signing for the distribution of medication to older persons living at home to contribute to reduced stress for UP and patient safety, this study indicates that a mapping of resources may be necessary prior to implementation. It is also important to ensure that there is good coverage for the smartphone devices and to establish instructions for UP on what to do in the case of downtime. Education and information were considered insufficient by the UP. Therefore, it is important that UP receive pre-implementation education and are involved in the entire implementation process. As the implementation of e-health technologies may provide a new way for UP to perform their duties, policies and procedures might need to be reviewed. It is also important to inform and involve the older persons in the introduction of digital signing. The result reveals the need for four preparatory steps before implementation of a digital medication signing list for UP, namely; education, work situation, technology and care relationship. The prepatory steps are illustrated in Figure 1 .

This study can contribute to a deeper understanding of the experiences of UP when implementing digital signing lists via a smartphone application for distribution of medication in municipal healthcare. The study also explores the conditions under which RNs delegate medication administration to UP. The UP need both skills to administer medication and technical skills to ensure patient safety. The challenge of remembering the password for the app as well as to sign it was also revealed. 


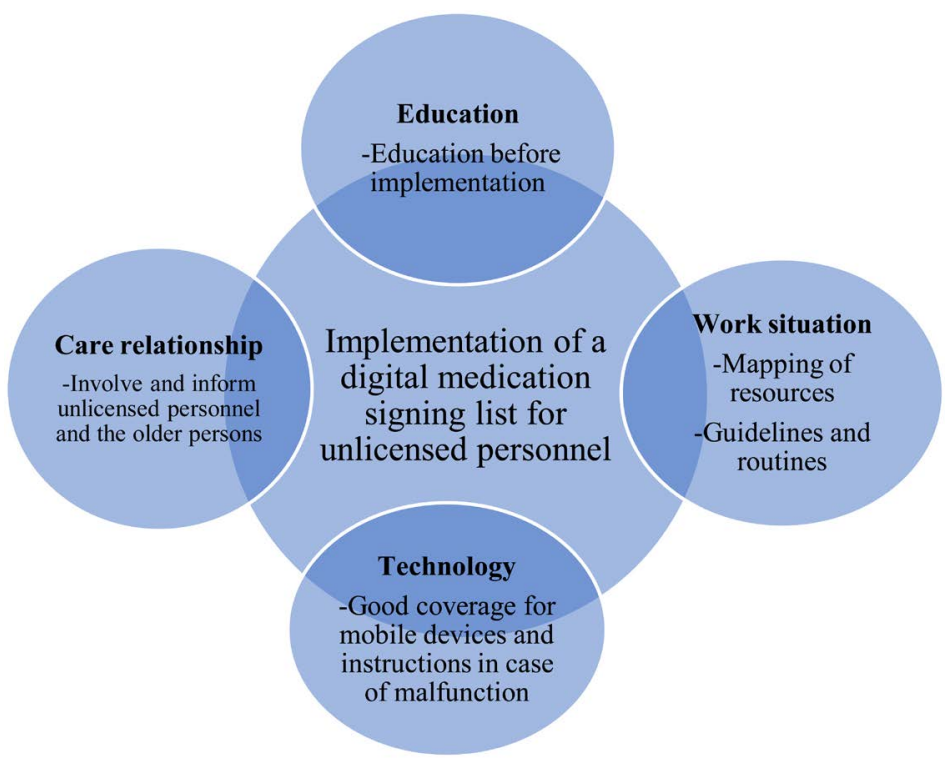

Figure 1. Illustration of the preparation required before the implementation of a digital medication signing list for UP.

\section{Limitations}

A number of limitations should be considered. The fact that the study solely included people willing to share their experiences of digital signing lists via a smartphone application for medication distribution means that the findings should be interpreted with caution. Another limitation is that only $33 \%$ of the invited UP returned the questionnaire. While more participants would have been preferable, the study nevertheless provides a first impression of digital signing lists in municipal healthcare. As the responses to the questionnaire contained a high degree of generalization, the next step was to explore the meaning of the different experiences through an interview study. The fact that 20 UP participated in the interview study is a strength.

To enhance the reliability, the interviews were transcribed verbatim, the text was coded, and the research group continuously discussed the subthemes and themes throughout the analysis process [17]. The opportunity for the authors to compare their interpretations of the text enhances the study's validity, as does the fact that the statements obtained through the questionnaires and individual interviews were found to be consistent with each other.

Obviously, the fact that the results are based on statements from personnel working in one Swedish healthcare region raises questions about the generalizability of the findings. To strengthen the quality of the analysis, the authors have provided examples of contextual details to help readers assess the transferability of the findings to other settings. The volume of material collected determines whether the data provide enough patterns and themes related to the phenomenon under study. Future research in this field would certainly benefit from larger samples gathered from multiple healthcare settings.

All of the present authors have worked as nurses. Their nursing background 
can be seen both as an advantage and a disadvantage, as on the one hand it improved their understanding of the participants' experiences, while on the other their preunderstanding may cause them to unknowingly influence the participants during interviews and/or to over-interpret the data during the analysis phase. To avoid or at least minimize these risks, the authors used an interview guide and attempted to maintain a critical attitude throughout the analysis process.

This study can contribute useful information about digital signing lists using smartphone applications for the distribution of medication. We believe that the results can be transferred to other healthcare systems, particularly those with similar routines and structures for the planning of coordinated care.

\section{Conclusions}

Technological developments will lead to major changes in the content of the UP's work, requiring them to gain additional professional skills. It is essential that UP feel safe when using new technology, as otherwise it can affect their work in municipal healthcare. In order to improve UP's professional skills it is important to include them at an early stage in the implementation of new technologies, inform them by means of education, guidelines and routines and enable them to have regular meetings with RNs, thus giving them an opportunity to discuss any problems experienced with the new technology. In this way, UP have the opportunity to develop technological skills.

The UP in municipal healthcare perceive being delegated tasks, such as the distribution of medication using digital signing lists via a smartphone application, as a responsibility. Digital signing in the smartphone made work easier when everything functioned as intended. The UP felt that the application was more transparent than paper records in terms of what patients needed help with. Instructions for various parts of the application that the UP could easily look up and follow gave them a sense of security at work. The disadvantage of digital signing lists was that the UP felt that the smartphone took up much of their attention when they were with the patient. They also believed that digital signing lists made patients less involved in their own care. This study may help nurses to gain a better understanding of UP experiences of the introduction of digital signing and responsibility for administering medication in the patient's own home.

\section{Funding Statement}

This research received no specific grant from any funding agency in the public, commercial or non-profit sectors.

\section{Author's Contributions}

SP and MK: Study design and manuscript preparation. All the authors (SP, CK and $\mathrm{MK}$ ) have contributed and agreed on the final version of the manuscript. 


\section{Acknowledgements}

The authors acknowledge the participants for sharing their experiences of the topic and Monique Federsel for reviewing the English language.

\section{Ethical Approval}

The regional ethical review board approved the study (Dnr 932-18), which is a prerequisite for ethically acceptable and reliable scientific research in accordance with the Declaration of Helsinki (2018).

\section{Data Availability Statement}

The data that support the findings of this study are available from the corresponding author upon reasonable request.

\section{Conflicts of Interest}

No potential conflict of interest was reported by the authors.

\section{References}

[1] National Board of Health and Welfare (2019) eHealth and Welfare Technology in Municipalities. Follow-Up of Developments in eHealth and Welfare Technology in Municipalities. (In Swedish) https://www.socialstyrelsen.se/

[2] State Public Reports (2020) The Technology of the Future in the Service of Care. Report of the Inquiry into Welfare Technology in Older Care. State Public Reports, Stockholm. (In Swedish)

https://www.regeringen.se/494156/contentassets/576aa4588db340b0ad052537ae905 $11 \mathrm{~d} /$ framtidens-teknik-i-omsorgens-tjanst-sou-2020_14.pdf

[3] HSLF-FS (2017) Swedish National Board of Health and Welfare Regulations and General Advice on Medications Handling in Healthcare. National Board on Health and Welfare, Stockholm. (In Swedish)

[4] Swedish Government (2010) Patient Safety Act. (In Swedish) https://www.riksdagen.se/sv/dokument-lagar/dokument/svensk-forfattningssamlin g/patientsakerhetslag-2010659_sfs-2010-659

[5] Gransjön Craftman, A., Hammar, L.M., von Strauss, E., Hillerås, P. and Westerbotn, M. (2015) Unlicensed Personnel Administering Medications to Older Persons Living at Home: A Challenge for Social and Care Services. International Journal of Older People Nursing, 10, 201-210.

[6] Hylte Municipality. (In Swedish) https://www.hylte.se/

[7] Research Institutes of Sweden. (In Swedish) https://www.ri.se/sv/fallstudier/digital-signering

[8] Rydenfält, C., Persson, J., Erlingsdottir, G. and Johansson, G. (2019) eHealth Services in the Near and Distant Future in Swedish Home Care Nursing. CIN: Computers, Informatics, Nursing, 37, 366-372. https://doi.org/10.1097/CIN.0000000000000536

[9] Skär, L. and Söderberg, S. (2018) The Importance of Ethical Aspects When Implementing eHealth Services in Healthcare: A Discussion Paper. Journal of Advanced Nursing, 74, 1043-1050.

[10] Öberg, U., Orre, C.-J., Isaksson, U., Schimmer, R., Larsson, H. and Hörnsten, Å. 
(2018) Swedish Primary Healthcare Nurses' Perceptions of Using Digital eHealth Services in Support of Patient Self-Management. Scandinavian Journal of Caring Sciences, 32, 961-970.

[11] Nakrem, S., Solbjør, M., Nilstad Pettersen, I. and Hestvik Kleiven, H. (2018) Care Relationships at Stake? Home Healthcare Professionals' Experiences with Digital Medicine Dispensers-A Qualitative Study. BMC Health Services Research, 18, Article No. 26. https://doi.org/10.1186/s12913-018-2835-1

[12] National Board of Health and Welfare (2017) Patient Säkerhet. National Board of Health and Welfare, Stockholm. (In Swedish) https://patientsakerhet.socialstyrelsen.se/om-patientsakerhet

[13] World Health Organization (2011) WHO Multi-Professional Patient Safety Curriculum Guide. World Health Organization, Geneva.

https://www.who.int/patientsafety/education/mp_curriculum_guide/en/

[14] World Health Organization (2009) Human Factors in Patient Safety: Review of Topics and Tools. Geneva.

https://apps.who.int/iris/bitstream/handle/10665/252273/9789241511612-eng.pdf

[15] Khalil, H. and Lee, S. (2018) Medication Safety Challenges in Primary Care: Nurses' Perspective. Journal of Clinical Nursing, 27, 2072-2082.

[16] Metlay, J.P., Cohen, A., Polsky, D., Kimmel, S.E., Koppel, R. and Hennessy, S. (2005) Medication Safety in Older Adults: Home-Based Practice Patterns. Journal of the American Geriatrics Society, 53, 976-982.

[17] Lundman, B. and Hällgren Graneheim, U. (2017) Kvalitativ Innehållsanalys. In: Höglund Nielsen, B. and Granskär, M., Ed., Tillämpad Kvalitativ Forskning Inom Hälso-och Sjukvård, 3rd Edition. Studentlitteratur, Lund, 219-234. (In Swedish)

[18] Polit, D.F. and Beck, C.T. (2012) Nursing Research: Generating and Assessing Evidence for Nursing Practice. 9th Edition, Wolters Kluwer Health/Lippincott Williams \& Wilkins, Philadelphia.

[19] Health and Medical Services Act (2017). (In Swedish) https://lagen.nu/2017:30\#K2P6S1

[20] Trost, J. (2012) Enkätboken. Studentlitteratur, Lund. (In Swedish)

[21] Gransjön Craftman, Å., Grape, C., Ringnell, K. and Westerbotn, M. (2016) Registered Nurses' Experience of Delegating the Administration of Medicine to Unlicensed Personnel in Residential Care Homes. Journal of Clinical Nursing, 25, 3189-3198.

[22] De Raeve, P., Gomez, S., Hughes, P., Lyngholm, T., Sipilä, M., Kilanska, D., Hussey, P. and Xyrichis, A. (2017) Enhancing the Provision of Health and Social Care in Europe through eHealth. International Nursing Review, 64, 33-41.

[23] Spånt Enbuske, A. (2019) Välfärdsteknik för Trygghet, Hälsa och Utveckling i Arbetet. (In Swedish)

https://www.kommunal.se/sites/default/files/valfardsteknik_for_trygghet_halsa_och _utveckling_i_arbetet_kommunal_2019_webb.pdf 